\title{
CREB Protein Expressed Differently in the Frontal Cortices of Datura stramonium Treated Rats: Implication for Addiction and Neurodegeneration
}

\author{
Peter E. Ekanem ${ }^{1}$, Dare S. Sunday ${ }^{2}$, Kidanemariam Gaim ${ }^{1}$ \\ ${ }^{1}$ Anatomy Unit, Institute of Biomedical Sciences, College of Health Sciences, Mekelle University, Mekelle, \\ Ethiopia \\ ${ }^{2}$ Anatomy Department, Faculty of Biomedical Sciences, Kampala International University, Kampala, Uganda \\ Email: ‘etimakpan@gmail.com, daresamdare@gmail.com, kidegaim@yahoo.com
}

Received 11 April 2016; accepted 10 May 2016; published 13 May 2016

Copyright $(2016$ by authors and Scientific Research Publishing Inc.

This work is licensed under the Creative Commons Attribution International License (CC BY). http://creativecommons.org/licenses/by/4.0/

(c) () Open Access

\begin{abstract}
Background: cAMP response element-binding protein (CREB) is one of the cellular transcription factors found in neurons. CREB is also important for the survival of neurons, and has an important role in the development of drug addiction. Datura stramonium (DS) is a tropical ubiquitous plant commonly used to increase the intoxication of certain beverages for recreational purposes. The seeds of this plant are very toxic and may produce addiction on prolong usage. This research investigated the effects of administration of high doses of DS seeds on the expression of CREB protein in both male and female rats' frontal cortices and its implication in addiction and neurodegeneration. Materials and Methods: The study was conducted with a total of 24 male and female Wistar rats weighing $200 \mathrm{~g}-250 \mathrm{~g}$. The rats were divided into three groups of 8 rats each. Each group was further divided into four sub-groups of 2 rats each. Ethanolic dried seed extract of DS was diluted in normal saline and administered intraperitoneally (i.p.) to the treatment groups. The treated sub-groups received $750 \mathrm{mg} / \mathrm{kg}$ of DS extract once in group 1, twice in group 2 and thrice in group 3 daily for 4 weeks respectively, while the control sub-groups received i.p. normal saline concurrently for the same duration of time. The rats were euthanized and an analysis of variance (ANOVA) was computed to detect a significant main difference of DS effect on CREB expression for each group, while post hoc Bonferroni Test compared CREB protein expression between male and female groups. Result: There were significant differences in the expression of CREB protein between the sub-groups and between the male and female rats of treated sub-group $(p<0.05)$ compared to the controls. There was a decrease in the female treated sub-groups and an increase in the male treated sub-groups compared to the respective controls. Conclusion: High
\end{abstract}

${ }^{*}$ Corresponding author.

How to cite this paper: Ekanem, P.E., Sunday, D.S. and Gaim, K. (2016) CREB Protein Expressed Differently in the Frontal Cortices of Datura stramonium Treated Rats: Implication for Addiction and Neurodegeneration. Journal of Behavioral and Brain Science, 6, 209-218. http://dx.doi.org/10.4236/jbbs.2016.65021 
doses of $D S$ administration for a prolong time may affect the expressions of CREB protein differently in male and female treated rats which may consequently lead to addiction and neurodegeneration affecting frontal cortex neurons.

\author{
Keywords \\ CREB Protein, Datura stramonium, Wistar Rats, Frontal Cortices' Neurons, Neurodegeneration, \\ Addiction
}

\title{
1. Introduction
}

One of the cellular transcription factors found in neurons is CREB (cAMP Response Element-Binding Protein). CREB is a member of a basic leucine zipper (bZIP) CREB/ATF-1 sub-family which includes members such as CREM (cAMP Responsive Element Modulatory protein) and ATF-1 (Activating Transcription Factor 1), both of which bear high bZIP region sequence homology [1] and can bind as homo-or hetero-dimers with these members of cAMP Responsive Element (CRE) sequence via the leucine zipper in the promoter regions of target genes [2] [3].

CREB protein also has an important role in the development of drug addiction and in the survival of neurons [4]. Various immunoblotting data have shown that CREB protein or activated CREB is regulated by various drugs of abuse within brain regions implicated in addiction. These data raise the question as to the precise role of CREB in mediating addiction-related behaviours [5].

Studies have also shown other intracellular signal transduction pathways culminating in the activation of CREB, as well as various paradigms of drug abuse which activate this key molecular substrate. Some of the intracellular signaling transduction pathways implicated in the activation of CREB demonstrated include: Gprotein, ion channel and growth factor drug receptor targets [5]. These pathways are known to often involve the association of a drug ligand with its respective receptor target, and a kinase phosphorylation cascading pathway which culminates in the translocation of a second messenger into the nucleus, phosphorylating CREB at Ser ${ }^{133}$ into pCREB [5]. Increasing evidence has supported the contribution of this nuclear transcription factor, CREB, in the development of addiction, both in contribution to phenotype and expression in brain regions critical to various aspects of drug-seeking behaviour and drug reward [5]. According to Blendy and Maldonado (1998) [2], a considerable amount of evidence is accumulating which implicates alterations in several components of the cAMP signal transduction cascade in these drug-induced processes.

Addiction has been defined as the compulsive or uncontrolled use of a drug, [6] which develops after repeated drug exposure, despite severe adverse consequences [7] [8]. It is said to be the progression from recreational or controlled use of a drug to this unbalanced, compulsive state that is a distinguishing hallmark of addiction. The underlying pathology of addiction has been partly described by different theories, and prominent among these is the opponent process theory [6]. Another theory is an incentive salience model which explains excessive drug wanting [9] and the development of learned adaptations, describing drug-memory associations [10]. The latter theory might explain the reason why people crave for such drugs despite their adverse effects to the brain. $D a-$ tura stramonium (DS) might fall into the category of these drugs because of its recreational usage and hallucination property.

Datura stramonium is a tropical plant which has wide distribution and usage and is often used recreationally as previously noted in certain cultures. It is a typical example of a hallucinogen with wide and varied uses. Some medicinal uses of the plant may include: anti-inflammatory (a property found in all parts of the plant), and stimulation of the central nervous system among others. It is used in alcoholic beverages to increase intoxication and smoked in like manner as tobacco due to its psychoactive properties. Cases of poisoning have been reported after eating the DS plant [10]. Death may occur from heart failure after ingesting 125 seeds because the seeds contain the highest concentration of tropane alkaloids in the plant. The wide distribution, strong toxicity and potential for occurrence in foodstuffs are responsible for the numerous incidents of toxicity in humans [10]. The range of toxicity of the $D S$ seed is highly variable and unpredictable because the concentration of specific alkaloids varies with species, cultivation, environment, temperature, moisture and storage [11]. This contributes to the danger of its misuse and addiction [12]. Historically, various behavioural patterns have been associated with 
excessive consumption of DS. Male and female animals have also been shown to respond differently to the treatment of $D S$ plant. In many cases investigated, the female animals were observed to be more affected than the males [10].

Earlier work carried out to determine which regions of the brain are critically involved in addiction and subsequently, what pathways are consistently activated, identified certain areas of the brain and pathways as substrate for certain forms of drug addiction. Studies employing both lesioning experiments [13] [14] and pharmacological manipulations [15] [16] have collectively demonstrated the importance of certain structures including the prefrontal cortex (PFC) of the frontal lobe, nucleus accumbens (NAcc), dorsal striatum, hippocampus and amygdaloid nuclei in relation to drug-seeking behaviours and drug-induced plasticity. The frontal cortex has been majorly used for such studies because it contains network of neurons as the substrate for functions that include: comprehension, cognition, communication, reasoning, problem-solving, abstraction, imagination, planning and memory.

The frontal cortex includes the prefrontal cortex, the motor cortex, frontal eye field, Broca's area of speech and other functional areas of the frontal lobe. The grey matter of the cerebral cortex is generally a convoluted, layered sheet of tissue, 2 - $3 \mathrm{~mm}$ thick in man but with a surface area of several hundred square $\mathrm{cm}$ [17]. Like other parts of the cerebral cortex, the frontal cortex generally consist of six layers: layer 1) is the most superficial layer with many dendritic and axonal processes and few cell bodies, layer 2) contains many small neurons with processes which established intracortical connections and layer 3) contains medium-sized neurons whose processes give rise to association and commissural fibres. Layer 4) serves as the site of termination of afferent fibres from different thalamic nuclei, layer 5) is the site of the origin of projection fibres to extra cortical targets such as the basal ganglia, thalamus, brain stem and spinal cord (in the primary motor cortex, this layer contains the giant Betz cells, which project fibres into the pyramidal tract), and layer 6) also contains association and projection neurons [18].

Experimental analyses have revealed that cells in the frontal lobe are significantly more spinous than those in the other lobes of the cerebral cortex [19] [20]. The numerous spines from the neurites of this part of the brain have been correlated with its integrative role in behaviour. Drug or factor that affects the frontal cortex neurites structure may also affect the neuronal structure and ultimately alter its integrative role in behaviour.

We hypothesized in this work that, prolonged use of DS seeds in high doses may alter the CREB protein transcription activity and affect its levels in the neurons of the frontal cortex. Also, that CREB protein may be expressed differently since male and female rats have been shown to respond differently to the administration of this drug. These alterations may lead to addictive behavioural patterns and neurodegeneration in the frontal cortex neurons of the treated rats.

\section{Materials and Methods}

\subsection{Plant Material Handling}

Datura stramonium seeds harvested in Samaru, Zaria, Nigeria and identified in the herbarium of Biological Sciences, Ahmadu Bello University (A.B.U.) and the Department of Pharmacognosy, Faculty of Pharmaceutical Sciences of the same university were used for this work. The seeds were air dried and extracted with 96.5\% ethanol. Phytochemical analysis identified the chemical composition of the extract. Dragendorff's reagent confirmed the presence of the major alkaloids and thin layer chromatography (TLC) was used to identify the presence of scopolamine, hyoscine, atropine and hyoscyamine as the major alkaloids.

\subsection{Animal Preparation for Experimentation}

A total of 24 male and female healthy adult Wistar rats, weighing $200 \mathrm{~g}$ - $250 \mathrm{~g}$ were randomly selected and used in this study. The animals were obtained from the animal house of the molecular biology unit of Veterinary Department, Makerere University, Uganda. The University's rules and regulations governing the number of non-human animals and handling of these animals for multi-purpose research were adhered to. This work therefore, was conducted in accordance with ethical committee guidelines on animal handling of the university.

The experimental rats were randomly selected and assigned to three groups of 8 animals each which was made up of males and females. Each group was sub-divided into four sub-groups of A, B, C and D of 2 animals each. Sub-groups A and B were made up of control and treated male rats respectively, while C and D contained female control and treated rats respectively. The control sub-groups received normal saline i.p. for 4 weeks 
while the treated sub-groups received DS seed extract diluted in normal saline i.p. for 4 weeks. Bania et al. (2004) [21] proposed a pretreatment dose of $7.5 \mathrm{mg} / \mathrm{kg}$ atropine equivalent of $D S$ seed extract as a protective agent in severe organophosphate toxicity. This was used as a baseline for the calculation of the treatment doses. To induce intoxication, $7.5 \mathrm{mg} / \mathrm{kg}$ was increased tenfold since the $\mathrm{LD}_{50}$ conducted on this specie of $D S$ was found to be above $5000 \mathrm{mg} / \mathrm{kg}$. Sub-groups B and D of each group were treated with different doses of DS extract. Group 1 received $750 \mathrm{mg} / \mathrm{kg}$ i.p once daily, the second group received $750 \mathrm{mg} / \mathrm{kg}$, i.p twice daily and group 3 received $750 \mathrm{mg} / \mathrm{kg}$, i.p thrice daily. The animals were fed with pellets and tap water and were observed daily after administration for signs that may arise from $D S$ administration. The animals were thereafter sacrificed by decapitation, their brains were dissected and the frontal cortices of both male and female rats carefully removed.

\subsection{Preparation of Brain Extract and Western Blot Analysis of CREB Protein}

$0.9369 \mathrm{~g}$ of each of the frontal cortices of both male and female rats was placed in a centrifuge tube containing 2 mls of PEME solution (1 mM PIPES, 0.1 mM EDTA, 1 mM EGTA, 1 mM PMSF, Tris-choride, acrylamide and $0.1 \%$ protease inhibitor) in an ice block tray. A cocktail tablet (Roche Co) was added to further inhibit the action of other proteases [22]. The brain sections and buffer were poured into a Petri dish and chopped into pieces. Brain pieces were homogenized using a sonicator machine (MSE), and spinned with a micro centrifuge at $100,000 \mathrm{rpm}$ at $4^{\circ} \mathrm{C}$ for 10 minutes (Sorvail RF Micro centrifuge). The clear supernatant was extracted with a micropipette, dispensed into smaller glass tubes and stored at $-80^{\circ} \mathrm{C}$ overnight. Two gels were prepared: one gel for staining with Coomasie blue and the other for Western blot. The gels were loaded at the same time to give the same experimental conditions. Protein concentration was determined using bicinchonic acid assay (BCA) kit (Pierce Biotechnology). A total of $40 \mu \mathrm{g}$ of total protein from each sample was applied for Western Blot. Protein samples were denatured and dissolved in 8\% SDS-PAGE then transferred to a nitrocellulose membrane. After the transfer, the membrane was removed from the sandwich and rinsed in distilled water. The membrane was stained for 30 seconds with ponceaus stain to verify the effectiveness of the transfer. It was immediately washed three times in $0.1 \%$ Tween 20 in phosphate buffer saline (PBS). The membrane was then placed in a glass plate and blocked at room temperature in a blocking solution (Bovine serum albumin, tween 20, PBS and 5\% nonfat milk) and incubated overnight at $4^{\circ} \mathrm{C}$. The blocking solution was poured away and the membrane washed three times with PBS. The primary antibody CREB-1 (C-21) rabbit polyclonal antibody (SC-186-Santa Cruz Bio technology) was added at a dilution of 1:200 according to the manufacturer's recommendation and allowed to incubate for 1 hour at room temperature with gentle agitation.

Removal of unbound primary antibody was done by washing the membrane in PBS three times at room temperature. The secondary antibody i.e. Goat—rabbit $1 \mathrm{gG}$ coupled to horse radish peroxidase (sigma) was diluted 1:10,000 in antibody solution. The membrane was then incubated in a rocking platform for one hour at room temperature.

The membrane was developed in hydrogen peroxide (Sigma co.) and the blot on the membrane captured in Enhanced Chemiluminescence (New Life Science Products, Boston, MA). Films were exposed for 10 seconds and developed. Protein levels were normalized before immunoprecipitation by DC assay (Bio-Rad) and GAPDH was chosen as an internal control protein because of its lower molecular weight and expressivity in all groups.

\subsection{Statistical Analysis}

The protocol used in analyzing the intensity of the protein bands in the western blot was image J. Analysis of variance (ANOVA) was computed to detect a significant main effect of $D S$ effect on CREB expression in each group, while post hoc Bonferroni Test for CREB protein expression between the groups was used to compare the differences between male and female groups.

The data was presented in percentage, with the profile plot representing the relative density of the content over each lane. The results from the package were copied to Microsoft excel sheet and represented in graphs, $\mathrm{P}$ was considered significant at $\leq 0.05$.

\section{Result}

24 adult healthy male and female wistar rats were selected and used in this experiment. The animals were randomly assigned into three groups and each group was subdivided into 4 sub-groups which received normal saline as 
control and treated sub-groups which received DS seed extract.

SDS-PAGE bands and the marker were used both in the treated and control sub-groups to identify the CREB protein at $43 \mathrm{KDa}$ while GAPDH at $37 \mathrm{KDa}$ served as internal control for CREB protein expression as seen in Figure 1. CREB protein band at $43 \mathrm{KDa}$ indicated by Western blot showed increased intensity of expression in all the male treated sub-groups compared to the controls as seen in Figure 1(b) and decreased intensity of expression in female treated sub-groups compared to the controls (Figure 1(c)). Image J analysis of the CREB expression in the female treated sub-groups showed significant decrease in expression in all sub-groups compared to the controls (Figure 2(a)).

Analysis in males (Figure 2(b)) showed significant increase of expression in all treated sub-groups compared to the controls.

When the expressions of CREB protein in male and female treated sub-groups were compared, there was a significant difference between both sexes $(p<0.05)$; the treated males showed elevated CREB expression compared to the reduction in the treated females (2C). Post Hoc Bonferroni Test of CREB protein expression between groups showed that there were significant differences between female control groups (FC), female treatment groups (FT), male control groups (MC), and male treated groups (MT) as seen in Figure 2(d).

The physical signs of the effect of this drug were general weakness, lethargy, loss of weight and appetite towards the end of fourth week of treatment. However, no death was recorded.

\section{Discussion}

It is scientifically established that changes in gene expression underlie neural adaptations following exposure to drugs of abuse [10]. It is also widely believed that a key mechanism for regulating gene expression is through nuclear induction of the transcription factor CREB, implicated also in learning and memory. To investigate the effects of prolong high doses of $D S$ seed extract on the CREB protein expression in the frontal cortex neurons, we increased the doses, prolonged the administration of the extract to 4 weeks in male and female Wistar rats and compared these to the control sub-groups which received normal saline. There was significant increase in the expression of CREB in each treated male sub-group compared to the controls (Figure 1(b) \& Figure 2(b)). The female rats showed significant decrease in the expression of CREB in each treated sub-group compared with the controls $(p<0.05)$ as seen in Figure 1(c) \& Figure 2(a). When the expression of CREB in the males was compared with the females, there was a significant difference $(\mathrm{p}<0.05)$ between males and females. In males, CREB expression increased while in females it decreased (Figure 2(c) \& Figure 2(d)).

Ekanem et al. (2015) [23] reported alterations of CREB protein in the hippocampal neurons of male and female rats after administration of high doses of DS. The changes in the expression of CREB in this work may

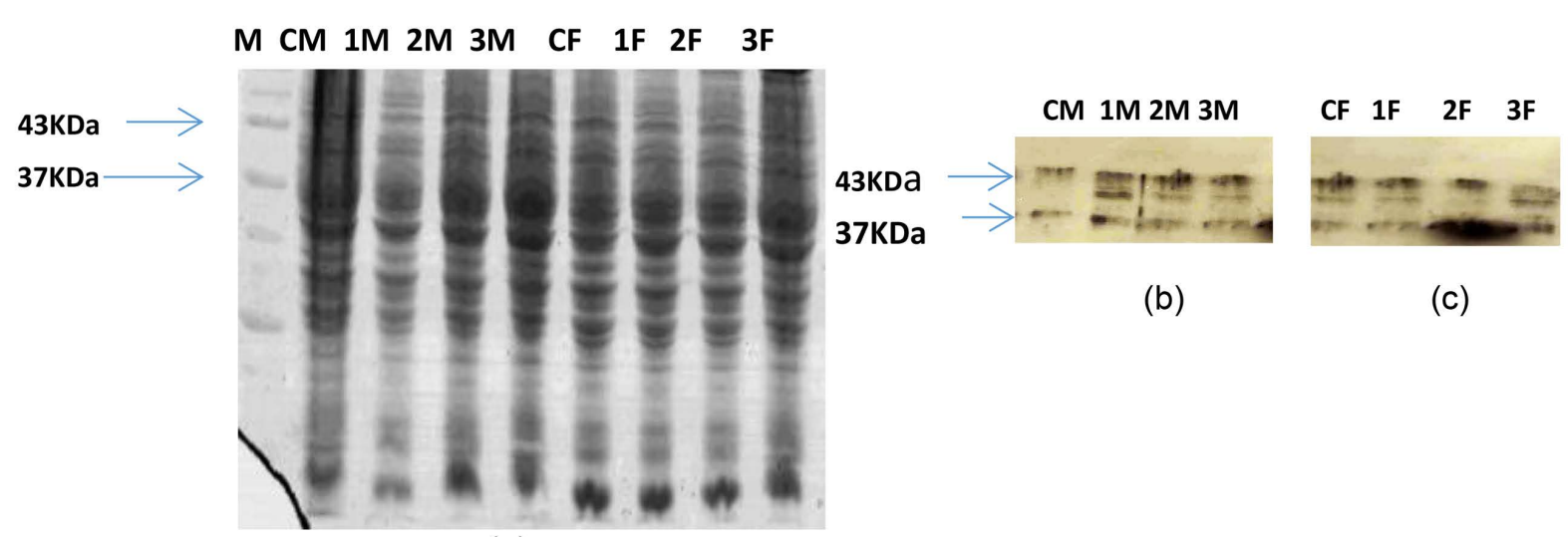

(a)

Figure 1. Expression of CREB protein in both male and female wistar rats. (a) SDS-PAGE with a marker indicating the CREB protein at $43 \mathrm{KDa}$ and internal control protein $\mathrm{GAPDH}$ at $37 \mathrm{KDa}$. $(\mathrm{M}=$ marker, $\mathrm{CM}=$ control male, $1 \mathrm{M}=$ group 1 treated male, $2 \mathrm{M}$ = group 2 treated male, $3 \mathrm{M}=$ group 3 treated male; $\mathrm{CF}=$ control female, $1 \mathrm{~F}=$ group 1 treated female, $2 \mathrm{~F}=$ group 2 treated female and $3 \mathrm{~F}$ = group 3 treated female. (b) Western blots indicating CREB protein at $43 \mathrm{KDa}$ and GAPDH at $37 \mathrm{KDa}$ in male rats $(\mathrm{CM}=$ control male, $1 \mathrm{M}=$ group 1 treated male, $2 \mathrm{M}=$ group 2 treated male, $3 \mathrm{M}=$ group 3 treated male). (c) Western blots indicating CREB expression at $43 \mathrm{KDa}$ and GAPDH at $37 \mathrm{KDa}$ in female rats ( $\mathrm{CF}=$ control female, $1 \mathrm{~F}=$ group 1 treated female, $2 \mathrm{~F}=$ group 2 treated female, $3 \mathrm{~F}=$ group 3 treated female). 


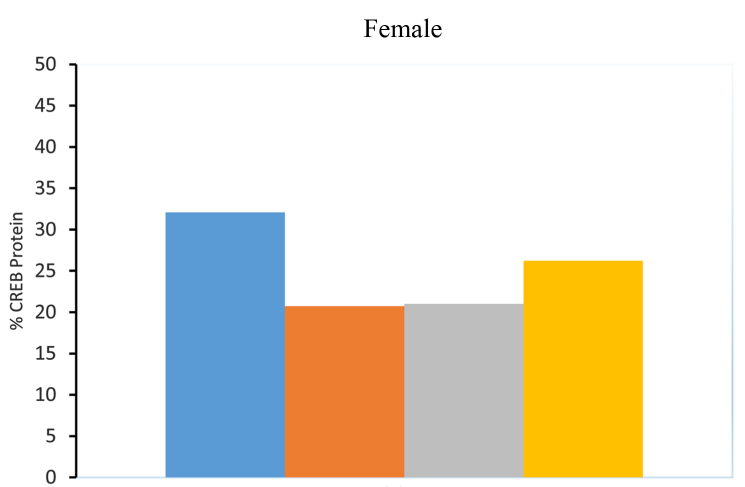

(a)

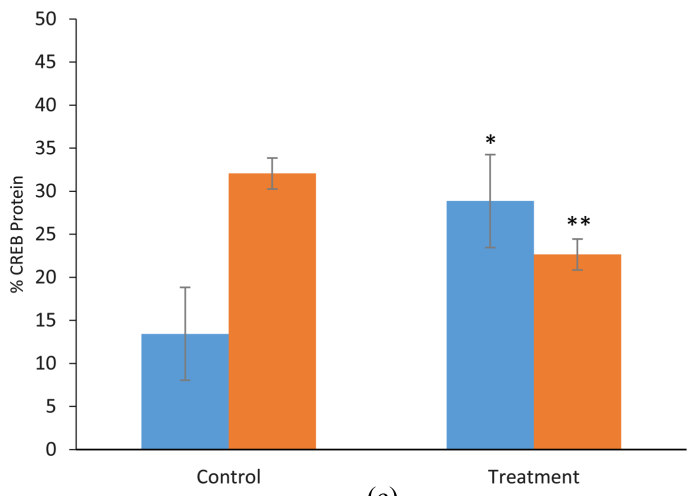

(c)

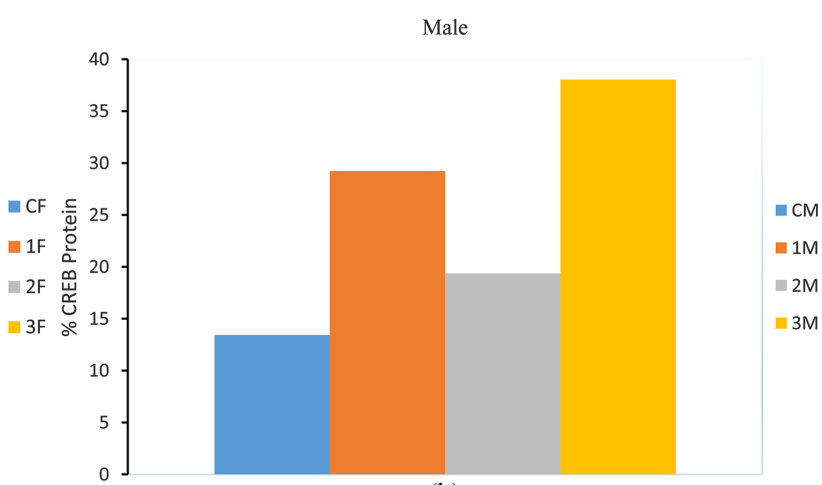

(b)

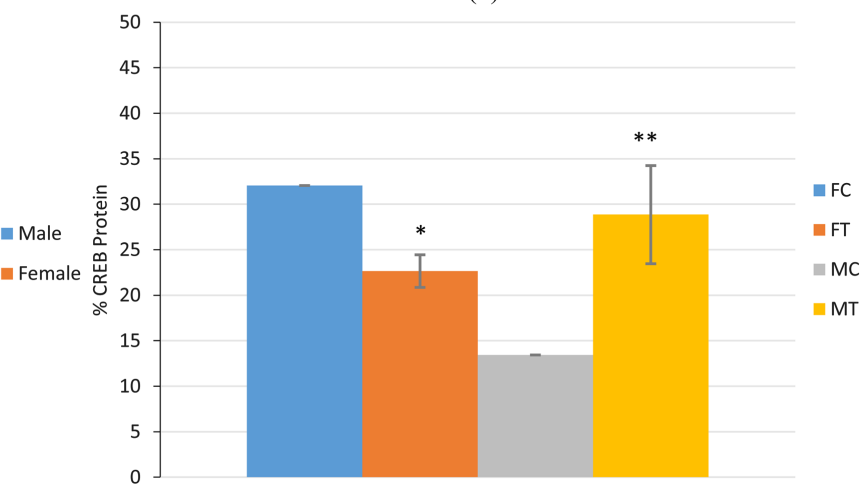

(d)

Figure 2. Graphical Representation of CREB protein expression in both Male and Female Wistar rats. (a) Percentage CREB protein expression level decreases in all treated female compared with the control. $\mathrm{CF}=$ Female Control group, $1 \mathrm{~F}=\mathrm{Female}$ treatment group 1, 2F = Female treatment group 2, 3F = Female treatment group 3. (b) Percentage CREB protein expression level increases in all treated male compared with the control. $\mathrm{CM}=$ Male Control group, $1 \mathrm{M}=$ Male treatment group $1,2 \mathrm{M}=$ Male treatment group 2, 3M = Male treatment group 3. (c) Comparison of the difference in CREB protein expression between control and treatment in both male and female groups was statistically significant $(\mathrm{p}<0.05)$ with significant increase in the male treatment compared to control male and significant decrease in female treatment compared to control female. (d) Post Hoc Bonferroni Test of CREB protein expression between groups showed that there were significant differences between Female control groups (FC), female treatment groups (FT), Male control groups (MC), and male treated groups (MT).

have been caused by prolonged administration of high doses of $D S$ seed extract. Scientific evidence has shown that drugs of abuse, administered repeatedly over time, cause physical dependence which is expressed by a withdrawal syndrome when the drug is removed from the system. These processes could be thought of as adaptations of the neuronal system to an altered pharmacological state [2]. Though the molecular mechanisms underlying these adaptations are still being investigated, a considerable amount of evidence is accumulating and this implicates alterations in several components of the cAMP signal transduction cascade in these drug-induced processes. The transcription factor cAMP response element binding protein (CREB) in particular has been shown both in vitro and in vivo to be altered in response to several drugs of abuse. It has been reported for example, that increase in growth hormone and its mediator insulin-like growth factor-I (IGF-I) induce neuroprotective effects and also stimulate neurogenesis [24] [25] when administered to opiate treated animals by mitigating the effects of opiates possibly through the transcription of CREB. Though, Ekanem et al. (2015) [23] reported increase in CREB in the hippocampal neurons of male and female rats after administration of high doses of $D S$, they also reported neurodegeneration of the hippocampal neurons. It seems that this increase did not show any form of neuroprotection or increase in the outgrowth of neurites as have been reported. The interpretation of these differences in expression may lie partly in the isoform of the CREB protein that may have been activated.

According to Bear et al. (2012) [18], there are two isoforms of CREB proteins: CREB-2 represses gene expression when it binds to the CRE; CREB- 1 activates transcription, but only when it is phosphorylated by protein kinase A (PKA). The question that may arise here is which of the CREB isoforms was actually elevated in $D S$ treated rats observed above. This calls for further investigation of which isoform of CREB protein could be elevated in DS treated male rats. But it has been observed that flies genetically engineered to over express the 
inactive form of CREB lose their ability to retain long-term memory [18]. The elevated CREB observed in the males may be as a result of over expression of CREB-2 or an inactive form of CREB protein, hence its inability to confer neuroprotection to neurons. This may induce neurodegeneration instead of neuroprotection to the neurons of the frontal cortex. If the above is true it means in male rats, $D S$ may have acted through a different pathway to produce an elevated CREB-2 isoform compared to female rats. The elevated CREB protein was not also uniform in the male treated groups as shown in Figure 2(b), where expression in $1 \mathrm{M}$ was higher than the expression in $2 \mathrm{M}$ compared to the control group. Though correlation analysis was not carried out to determine the doses of DS and the quantity of CREB protein expressed, this may be attributed to the tolerance level of this group to the $D S$ administration.

In the female treated sub-groups, there was significant CREB protein reduction within the treated groups as shown in Figure 2(a). This reduction has been generally associated with neurodegeneration in several areas of the brain studied. For example, neurodegeneration was observed when an opioid-induced attenuation of neurogenesis and apoptosis in hippocampus was demonstrated in male rats exposed to morphine [26]. This was attributed to decreased CREB protein expression in the treated rats. Dragana et al. (2009) [27] in their analysis of the hippocampus of A-CREB mice revealed a massive loss of neurons in the CA 1 subfield, most likely as a consequence of the dramatic and sustained inhibition of CREB expression and function in pyramidal neurons. They concluded that long term chronic inhibition of CREB function caused severe loss of neurons in the CA 1 subfield of the hippocampus. Studies conducted by Gao Ping-rui et al. (2014) [28] on the effects of cholecystokinin octapeptide (CCK-8) and its receptor antagonists on cAMP response element binding protein (CREB) and phosphorylated CREB (pCREB) expression in the frontal cortex and hippocampus of morphine withdrawal rats showed that CREB was expressed in both the cytoplasm and the nucleus. It was evidenced that morphine administration led to reduction in expression of CREB protein in the experimental rats. Since we have observed a decrease in the expression of CREB protein in DS treated female rats, it may imply that DS administration may induce neurodegeneration in the frontal cortex neurons as well and interfere with their integrative role.

An ever increasing body of evidence also implicates the molecular reduction of CREB protein in experimental paradigms related to addiction. These studies have provided a conflicting array of results regarding positive and negative regulation of pCREB expression. Also, divergences have been particularly noted across brain regions examined as well as the period of administration including: acute, chronic and subsequent to precipitated withdrawal [5]. It has been shown by various authors that either natural or precipitated withdrawal from drugs of abuse leads to a dramatic alteration in the expression of nuclear pCREB [5]. Substantial pCREB changes have also been observed following withdrawal from ethanol, morphine, psychostimulants, including amphetamine, methamphetamine and cocaine [29]-[33].

These data suggest that repeated exposure to drugs of abuse may produce sustained activation of intracellular transcription factors, resulting in the persistent and altered expression of functionally important gene products that may underlie the onset and maintenance of addiction.

Though the mechanism of DS seed alteration of the transcription factor is not known, it is believed that the toxins in DS seed alkaloids possess strong anticholinergic properties [34] which act as competitive antagonists to acetylcholine at peripheral and central muscarinic receptors at a common binding site [35]. As tertiary amines, these alkaloids also have central nervous system absorption, inhibit CVS receptors and result in a central anticholinergic syndrome of acute psychosis and delirium [34]. The changes in CREB protein expression observed in this work may therefore implicate $D S$ in the activation process of such transcription factors that may alter certain genes responsible for addiction.

Differences in expression of CREB in response to excitotoxic drugs as seen in this work in male and female rats have also been observed by several authors. Balschun et al. (2003) [36] showed in the adult mouse brain that loss of CREB protein neither prevents learning nor substantially affects performance in some hippocampus-dependent tasks. This implies either a species-specific or regionally restricted role of CREB in the brain or a compensatory upregulation of the cAMP response element modulator (CREM), or other as yet unidentified transcription factors [36]. A toxicological evaluation of DS seed extract was also conducted on male and female rats. The principal effects recorded were: decreased body weight, serum albumin and serum calcium, increased liver and testes weight (as a percentage of body weight), serum alkaline phosphatase and blood urea nitrogen. Female rats were found to show more marked responses to DS than males [37]. Thus, the differences between male and female treated rats' responses to the different administration of DS seed extract shown in this work may have been caused by the DS seed extract. 


\section{Conclusion}

It is evidenced from this work that DS may act through different pathways to alter the CREB protein expression in the frontal cortices of both male and female rats. This alteration of CREB protein transcription may affect the gene that expresses addiction and also trigger neurodegeneration. The consumption of DS seeds may need to be regulated by appropriate authorities as indiscriminate usage may contribute to or increase behavioral and mental retardations commonly seen in our society.

\section{Acknowledgements}

The authors would like to acknowledge the staff of Molecular Biology laboratory of Makerere University for permitting this work to be carried out in their laboratory and providing necessary technical support to the work. We also acknowledge, Mrs Regina Ekanem, Dr. Kendi Nyaga and Dr. Elizabeth Akika for their support and for going through this manuscript to correct typographical errors.

\section{Funding}

This work was primarily self-funded, but also received financial support from Kampala International University at later stages of the work.

\section{Authors' Contributions}

The corresponding author, Peter Etim Ekanem designed the conceptual, theoretical and contextual backgrounds of this work and took part in all parts of this work. Dare Sunday and Kidan Gaim analyzed the data, checked and tested the accuracy of the data interpretation.

\section{Competing Interest}

The authors declare that there is no competing interest.

\section{References}

[1] Shaywitz, A.J, Greenberg, M.E. (1999) A Stimulus-Induced Transcription Factor Activated by a Diverse Array of Extracellular Signals. Annual Review of Biochemistry, 68, 821-861. http://dx.doi.org/10.1146/annurev.biochem.68.1.821

[2] Blendy, J.A. and Maldonado, R. (1998) Genetic Analysis of Drug Addiction: The Role of cAMP Response Element Binding Protein. Journal of Molecular Medicine, 76, 104-110. http://dx.doi.org/10.1007/s001090050197

[3] Chao, J. and Nestler, E.J. (2004) Molecular Neurobiology of Drug Addiction. Annual Review of Medicine, 55, 113-132. http://dx.doi.org/10.1146/annurev.med.55.091902.103730

[4] Wang, Y., Ghezzi, A., Yin, J.C. and Atkinson, N.S. (2009) CREB Regulation of BK Channel Gene Expression Underlies Rapid Drug Tolerance. Genes, Brain and Behavior, 8, 369-376. http://dx.doi.org/10.1111/j.1601-183x.2009.00479.x

[5] Cameron, S.M. and Andrew, J.L. (2007) The Nuclear Transcription Factor CREB: Involvement in Addiction, Deletion Models and Looking Forward. Current Neuropharmacology, 5, 202-212. http://dx.doi.org/10.2174/157015907781695937

[6] Koob, G.F., Caine, S.B., Parsons, L., Markou, A. and Weiss, F. (1997) Opponent Process Model and Psychostimulant Addiction. Pharmacology Biochemistry and Behavior, 57, 513-521. http://dx.doi.org/10.1016/S0091-3057(96)00438-8

[7] Nestler, E.J. (1997) Molecular Mechanisms of Opiate and Cocaine Addiction. Current Opinion in Neurobiology, 7, 713-719. http://dx.doi.org/10.1016/S0959-4388(97)80094-3

[8] Koob, G.F., Sanna, P.P. and Bloom, F.E. (1998) Neuroscience of Addiction. Neuron, 21, 467-476. http://dx.doi.org/10.1016/S0896-6273(00)80557-7

[9] Kelley, A.E. and Berridge, K.C. (2002) The Neuroscience of Natural Rewards: Relevance to Addictive Drugs. The Journal of Neuroscience, 22, 3306-3311.

[10] Das, S., Grunert, M., Williams, L. and Vincent, S.R. (1997) NMDA and D1 Receptors Regulate the Phosphorylation of CREB and the Induction of c-fos in Striatal Neurons in Primary Culture. Synapse, 25, 227-233. http://dx.doi.org/10.1002/(SICI)1098-2396(199703)25:3<227::AID-SYN1>3.0.CO;2-D

[11] Goldfrank, L.R. (1994) Datura Plant. 5th Edition, Toxicological Emergencies, Appleton and Lange, Norwalk, $103-109$. 
[12] Haddad, L.M. and Winchester, J.F. (1990) Clinical Management of Poisoning and Drug Overdose. W.B. Saunders Co. New York, 345-350.

[13] Hiroi, N. and White, N.M. (1991) The Lateral Nucleus of the Amygdala Mediates Expression of the AmphetamineProduced Conditioned Place Preference. The Journal of Neuroscience, 11, 2107-2116.

[14] Dailey, J.W., Thomas, K.L., Howes, S.R., Tsai, T.H., Aparicio-Legarza, M.I., Reynolds, G.P., Everitt, B.J. and Robbins, T.W. (1999) Effects of Excitotoxic Lesions of the Rat Prefrontal Cortex on CREB Regulation and Presynaptic Markers of Dopamine and Amino Acid Function in the Nucleus Accumbens. European Journal of Neuroscience, 11, 1265-1274. http://dx.doi.org/10.1046/j.1460-9568.1999.00532.x

[15] Fuchs, R.A., Branham, R.K. and See, RE. (2006) Different Neural Substrates Mediate Cocaine Seeking after Abstinence versus Extinction Training: A Critical Role for the Dorsolateral Caudate-Putamen. The Journal of Neuroscience, 26, 3584-3588. http://dx.doi.org/10.1523/JNEUROSCI.5146-05.2006

[16] Vanderschuren, L.J, Di Ciano, P. and Everitt, B.J. (2005) Involvement of the Dorsal Striatum in Cue-Controlled Cocaine Seeking. The Journal of Neuroscience, 25, 8665-8670. http://dx.doi.org/10.1523/JNEUROSCI.0925-05.2005

[17] Stewart, S. (2007) Structure and Function of the Cerebral Cortex. Current Biology, 17, R443-R449. http://dx.doi.org/10.1016/j.cub.2007.03.044

[18] Bear, M.F., Connors, B.W. and Paradiso, M.A. (2012) Neuroscience: Exploring the Brain, Lippincot (Wolters Kluwer Health). Kindle Edition, Kindle Locations, 12791-12792.

[19] Guys, N.E. (2000) Pyramidal Cells of the Frontal Lobe: All the More Spinous to Think with. The Journal of Neuroscience, 20, 1-4.

[20] Elston, G.N., Tweedale, R. and Rosa, M.G.P. (1999) Cellular Heterogeneity in Cerebral Cortex: A Study of the Morphology of Pyramidal Neurons in Visual Areas of the Marmoset Monkey. Journal of Comparative Neurology, 415, 33-51. http://dx.doi.org/10.1002/(SICI)1096-9861(19991206)415:1<33::AID-CNE3>3.0.CO;2-M

[21] Bania, T.C., Chu, J., Bailes, D. and O’Neill, M. (2004) Jimson Weed Extract as Protective Agent in Severe Organophosphate Toxicity. Academic Emergency Medicine, 11, 335-338. http://dx.doi.org/10.1197/j.aem.2003.12.002

[22] Sambrook, J., Fritsch, E.F. and Maniatis, T. (1989) Molecular Cloning: A Laboratory Manual. 2nd Edition, Cold Spring Harbor Laboratory Press, New York, 1814-1875.

[23] Ekanem, P.E., Dare, S.S., Abba, S. and Tesfay, A. (2015) Hippocampal Pyramidal Cell Degeneration and Changes in CREB Protein in Datura stramonium Treated Rats. American Journal of Neuroscience, 6, 13-19.

[24] Isgaard, J., Abreg, D. and Nilsson, M. (2007) Protective Regenerative Effects of GH/IGF-1 Axis on the Brain. Minerva Endocrinologica, 32, 103-113.

[25] Nyberg, F. (2007) Growth Hormone and Brain Function. In: Ranke, M.B., Price, D.A. and Reiter, E.O., Eds., Growth Hormone Therapy in Paediatrics: 20 Years of KIGs, Karger, Basel, 450-460. http://dx.doi.org/10.1159/000101936

[26] Eisch, A.J., Barrot, M., Schad, C.A., Self, D.W. and Nestler, E.J. (2000) Opiates Inhibit Neurogenesis in Adult Rat Hippocampus. Proceedings of the National Academy of Sciences of the United States of America, 97, 7579-7584. http://dx.doi.org/10.1073/pnas.120552597

[27] Dragana, J.L., Mikel, A.M., Luis, V.O. and Roman, B.A. (2009) Inhibition of cAMP Response Element-Binding Protein Reduces Neuronal Excitability and Plasticity, and Triggers Neurodegeneration. Cerebral Cortex, 19, 2535-2547. http://dx.doi.org/10.1093/cercor/bhp004

[28] Gao, P.X., Ma, D., Wen, S., Yang, F., Yu, Z., Ni, S. and Li, C.M. (2014) Effects of CCK-8 and Its Receptor Antagonists on Expression of CREB and pCREB in Prefrontal Cortex and Hippocampus of Morphine Withdrawal Rats. Chinese Journal of Pathophysiology, 30, 1158-1165.

[29] Pandey, S.C., Roy, A. and Zhang, H.B. (2003) The Decreased Phosphorylation of Cyclic Adenosine Monophosphate (cAMP) Response Element Binding (CREB) Protein in the Central Amygdala Acts as a Molecular Substrate for Anxiety Related to Ethanol Withdrawal in Rats. Alcoholism: Clinical and Experimental Research, 27, 396-409. http://dx.doi.org/10.1097/01.ALC.0000056616.81971.49

[30] Chartoff, E.H., Papadopoulou, M., Konradi, C. and Carlezon, W.A. (2003) Dopamine-Dependent Increases in Phosphorylation of cAMP Response Element Binding Protein (CREB) during Precipitated Morphine Withdrawal in Primary Cultures of Rat Striatum. Journal of Neurochemistry, 87, 107-118. http://dx.doi.org/10.1046/j.1471-4159.2003.01992.X

[31] Hsieh, H.C., Li, H.Y., Lin, M.Y., Chiou, Y.F., Lin, S.Y., Wong, C.-H. and Chen, J.-C. (2002) Spatial and Temporal Profile of Haloperidol-Induced Immediate-Early Gene Expression and PhosphoCREB Binding in the Dorsal and Ventral Striatum of Amphetamine-Sensitized Rats. Synapse, 45, 230-244. http://dx.doi.org/10.1002/syn.10099

[32] Kuo, Y., Liang, K., Chen, H., Cherng, C., Lee, H., Lin, Y., Huang, A., Liao, R. and Yu, L. (2007) Cocaine-But Not Methamphetamine-Associated Memory Requires de novo Protein Synthesis. Neurobiology of Learning and Memory, 87, 93-100. http://dx.doi.org/10.1016/j.nlm.2006.06.004 
[33] McDaid, J., Graham, M.P. and Napier, T.C. (2006) Methamphetamine-Induced Sensitization Differentially Alters pCREB and Delta-FosB throughout the Limbic Circuit of the Mammalian Brain. Molecular Pharmacology, 70, $2064-$ 2074. http://dx.doi.org/10.1124/mol.106.023051

[34] Arnett, A.M. (1995) Jimson Weed (Datura stramonium) Poisoning. Clinical Toxicology Review, 18, 1-6.

[35] Winchester, H.L.M. (1990) Clinical Management of Poisoning and Drug Overdose. W.B. Saunders Co., New York, 345-350.

[36] Balschun, D., Wolfer, D.P., Gass, P., Mantamadiotis, T., Welzl, H., Schutz, G., Frey, J.U. and Lipp, H.P. (2003) Does cAMP Response Element-Binding Protein Have a Pivotal Role in Hippocampal Synaptic Plasticity and Hippocampus-Dependent Memory? The Journal of Neuroscience, 23, 6304-6314.

[37] Diker, D., Markovitz, D. and Rothman, M. (2007) Coma as a Presenting Sign of Datura stramonium Seed Tea Poisoning. European Journal of Internal Medicine, 18, 336-338. http://dx.doi.org/10.1016/j.ejim.2006.09.035 